\title{
Death in gene therapy trial raises questions about private IRBs
}

Osagie Obasogie took notice when he heard about the death of Jolee Mohr, who passed away on July 24 after participating in a gene therapy trial testing a rheumatoid arthritis treatment developed by Seattle-based Targeted Genetics. As director of the project on Bioethics, Law, and Society at the Center for Genetics and Society, a nonprofit organization based in Oakland, California, and as someone who has been studying US oversight of clinical trials, he felt the need to investigate.

So he did, and in mid-August, he

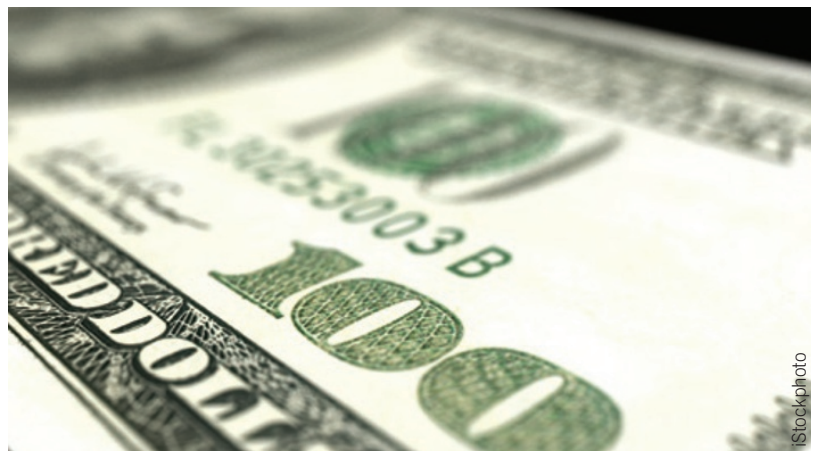

The increasing prevalence of for-profit institutional review boards is making some observers and bioethicists nervous.

private IRBs," says Jonathan Moreno, a bioethicist at the University of Pennsylvania in Philadelphia. "But I don't have any evidence that they do a worse job, and of course conflicts of interest come in many flavors." Not operating for a profit doesn't relieve an IRB of pressure-members of traditional IRBs may feel compelled to approve a trial that will bring substantial research funding and prestige to their institutions, for example. A 1998 audit of the IRB system by the Office of the Inspector General at the US Department of Health and Human Services found several examples in published a column in the Seattle Post Intelligencer highlighting possible concerns about the trial's design, in which he noted that "the institutional review board [IRB] charged with ensuring that the trials were conducted ethically is a for-profit enterprise also on Targeted Genetics' payroll." And he wasn't the only one suggesting that might be improper.

There is no hard evidence that Western Institutional Review Board, the "for-profit enterprise" that approved the trial, was remiss in its duties, and the US Food and Drug Administration (FDA) points out that IRBs handling clinical trials expected to be used for regulatory approval must follow FDA regulations no matter if they are for-profit or traditional. "An IRB is an IRB," says FDA spokeswoman Karen Riley. Regardless, the incident has turned a spotlight on for-profit IRBs, and the field makes many bioethicists instinctively uneasy.

In the US, IRBs have traditionally been run at the local level by individual research institutions, with the aim of enabling such bodies to provide ground-level oversight of experiments. But as the number of clinical trials grew, many traditional IRBs became overworked. The result was widespread delays in processing applications and concerns about whether the stretched IRB staff could adequately oversee ongoing trials. Today, researchers are increasingly turning to private, for-profit IRBs that promise quick turnaround times, especially for company-sponsored trials.

Although most feel that for-profit IRBs are rapidly expanding, there are no data about how their ranks have changed over the years, and no comprehensive list of how many such organizations exist-an indicator of how unregulated IRBs are. However, Western IRB, based in Olympia, Washington, is the oldest and largest private IRB in the US, and it has grown tremendously. In 1981, when the company first began charging for its services, it processed about 16 new applications, says company founder Angela

Bowen. Now, Western handles from 2,000-2,500 new applications each year. Chesapeake Research Review, a for-profit IRB based in Columbia, Maryland, earned \$5.5 million in 2004, finding a spot on Deloitte's list of the fastest-growing technology companies in North America.

Those types of numbers have ethics observers nervous. University of Minnesota bioethicist Carl Elliott says that when he tells people from other countries about the US's growing reliance on for-profit IRBs, the response is often an incredulous laugh. "It seems like an obvious conflict of interest for the people who are being regulated to be the sole funder of the regulator," he says. Critics of the system also raise concerns that a for-profit IRB could change its committees members so it is likely to approve protocols to please its customers. Trudo Lemmens, an associate law professor at the University of Toronto, says that he has been contacted by one former member of a for-profit IRB who claims to have witnessed the IRB doing precisely that.

But just as there is no comprehensive list of for-profit IRBs, there also is no system for comparing the quality of ethical review at for-profit and traditional IRBs. "That tells you something about the lack of oversight of the whole IRB structure," says Lemmens. Without those data, stories of poorly designed or mismanaged clinical trials overseen by for-profit IRBs can never rise above the anecdotal, especially in the face of traditional IRBs' own spotty record. "You might be able to cite a case or two" of misconduct at for-profit IRBs, says Ezekiel Emmanuel, a bioethicist at the National Institutes of Health. "But I can cite plenty of cases, too: Hopkins, Duke, the University of Colorado-all non-profits that have had their IRBs shut down at some time."

So although the Jolee Mohr death has brought forth critics of the for-profit IRB system, traditional IRBs carry hefty conflicts of their own. "In general there is an automatic suspicion about which hospital IRBs were housed in the offices of grants and contracts, "the very offices geared to bring in research dollars." Recently, some traditional IRBs have further blurred the line by directly charging investigators for their services as a way of defraying staff expenses.

Elliott also notes that a Canadian traditional IRB once overhauled committee membership after repeated rejections of a particular kind of placebo-controlled trial.

As for the Targeted Genetics trial itself, ethicists raised a number of concerns after Mohr's death. The consent form was too complex, some said, and Mohr's doctor - who was being paid to recruit subjects into the trial—should not have personally recruited one of his own patients. Although not an excuse, those flaws are found in many clinical trials. Adil Shamoo, a bioethicist at the University of Maryland School of Medicine in Baltimore, has read the Targeted Genetics 15page patient consent form and found no clear deficiencies, although he says it's common for subjects to misunderstand the risk they face when enrolling in a clinical trial.

"The risks were laid out in the consent form," says Shamoo, "but many human subjects are given this horrible, thick informed consent document and then choose to sign it right then and there."

That problem exists whether the IRB is traditional or for profit, and upon closer examination of the overall IRB landscape, for-profits may differ little from the flawed traditional system in the US. Bioethicist Arthur Caplan, also at the University of Pennsylvania, says that whether Western IRB rubber-stamped the Targeted Genetics approval deserves "some close scrutiny," but the important task ahead is to have "more of a debate than we've had about whether this [IRB structure] is the system we want."

Heidi Ledford, Boston 